\title{
Estudo dos Parâmetros de Polaridade Negativa na Soldagem MIG/MAG Polaridade Variável para Revestimento
}

\author{
Alexandre José Baumgaertner Filho ${ }^{1}$, Arnaldo Ruben Gonzalez ${ }^{1}$ \\ 1 Universidade Federal do Rio Grande do Sul - UFRGS, Laboratório de Soldagem \& Técnicas Conexas, Porto Alegre, RS, Brasil.
}

Recebido: 13 Fev., 2017

Aceito: 04 Maio 2017

E-mails: alexandre.baumgaertner@ufrgs. br (AJBF), ruben@mecanica.ufrgs.br (ARG)
Resumo: Este trabalho tem como objetivo a investigação dos efeitos dos parâmetros da polaridade negativa da curva de corrente no resultado da diluição dos cordões de solda no processo de soldagem MIG/MAG polaridade variável, visando buscar um conjunto de parâmetros que ofereça uma menor diluição, os quais são fundamentais para soldagem para revestimento. Através de um projeto de experimentos Box-Behnken com três fatores de controle (corrente negativa, tempo de corrente negativa e tempo de corrente de base), variando em três níveis cada um, foram depositados cordões de solda em aço inoxidável sobre aço ao carbono. Para esse processo foi atingido valores de diluição de $12 \%$ a 15\%, ideais para soldagem para revestimento. Foi verificado a significância do tempo de corrente negativa nos resultados da diluição, mostrando a oportunidade de utilização polaridade negativa no processo de soldagem MIG/MAG aplicado para revestimento.

Palavras-chave: MIG/MAG polaridade variável; Revestimento; Diluição; Aço inoxidável; Projeto Box-Behnken.

\section{Study of Negative Polarity Parameters in MIG/MAG Variable Polarity Welding for Cladding}

\begin{abstract}
This article aims to investigate the effects of the negative polarity parameters of the current curve on the result of the dilution of the weld beads in the MIG / MAG variable polarity welding process, targeting to find a set of parameters that offer a lower dilution, which is fundamental for welding for cladding. Through a design of experiments Box-Behnken with three control factors (negative current, negative current time and base current time) varying in three levels each, stainless steel weld beads were deposited on carbon steel. For this process, dilution values of $12 \%$ to $15 \%$ were achieved, ideal values for cladding welding. The significance of the negative current time in the dilution results was verified, showing the possibility of using negative polarity in the MIG/MAG welding process applied to the cladding.
\end{abstract}

Key-words: GMAW variable polarity; Cladding; Dilution; Stainless steel; Box-Behnken design.

\section{Introdução}

A técnica de soldagem MIG/MAG é um dos processos mais utilizados nos dias atuais. Segundo Nascimento e Vilarinho [1], o processo MIG/MAG foi patenteado em 1930 por Hobart e Devers, o qual foi chamado de GMAW (Gas Metal Arc Welding). Atualmente, esse processo é um dos mais utilizados no mundo, segundo Miranda e Ferraresi [2], isto é devido à alta produtividade, boa qualidade do cordão de solda e custo moderado do MIG/MAG.

Devido à demanda de aumento de produtividade e da necessidade da utilização de materiais mais leves na indústria, em conjunto com o avanço das tecnologias e a evolução dos processos de soldagem, notou-se avanços na técnica de soldagem MIG/MAG com corrente alternada e/ou polaridade variável.

Essa variante do processo MIG/MAG é encontrada com duas nomenclaturas na literatura atual, sendo elas: corrente alternada (CA) e polaridade variável (PV). Conforme Nascimento e Vilarinho [1], estas duas nomenclaturas são válidas, porém CA é mais utilizada para ondas de caráter senoidal e PV para pulsos de ondas quadradas, onde há variação brusca na transição da parte positiva à negativa, e vice-versa.

A polaridade negativa atualmente é empregada através de curvas de corrente com pulsos. Esta por sua vez, possui como principal benefício a alta taxa de fusão, proporcionando 
aumento na temperatura do eletrodo e por consequência minimiza os efeitos de temperatura no metal base. Conforme apresentado por Tong et al. [3], a utilização do eletrodo negativo comparado com o processo MIG/MAG convencional apresenta menores valores de temperatura na peça. Em decorrência, verificou-se uma redução da deformação da peça final, assim como uma menor penetração. Esse benefício também foi verificado por Park et al. [4], que constataram redução na temperatura da peça soldada para um aumento na utilização do eletrodo negativo.

A variação da taxa de fusão é uma das grandes limitações para a utilização do processo MIG/MAG com polaridade variável, pois gera uma grande instabilidade no processo de soldagem devido à alteração do comprimento do arco elétrico nas diferentes polaridades, como apresentado por Dutra et al. [5]. Conforme Puhl [6], a falta de estabilidade produz cordões de solda com qualidade abaixo do padrão aceitável, e é um dos principais fatores que tornam a técnica de corrente alternada na soldagem MIG/MAG pouco utilizada atualmente.

A corrente negativa fornece benefícios para a soldagem MIG/MAG, contudo não se pode utilizar apenas o eletrodo negativo, precisa-se do eletrodo positivo para estabilizar o arco e realizar a fusão da peça e do metal de solda, e por essa razão é utilizado uma curva de pulso para este tipo de processo, composta por corrente de pico positivo, corrente de base e corrente negativa, conforme a Figura 1.

A Figura 1 ilustra os parâmetros para definir a curva de corrente para utilizar a polaridade variável, os quais são: corrente de pico (Ip), tempo de pico (Tp), corrente de base (Ib), tempo de base (Tb), corrente negativa (In) e tempo de eletrodo negativo (Tn). Na soldagem é necessária a combinação de seis parâmetros, por isso os autores utilizam o termo percentual do eletrodo negativo (\%EN) para poder quantificar a variação da corrente negativa em relação à positiva e por sua vez, analisar os efeitos do eletrodo negativo no processo de soldagem. Este termo \%EN é encontrado na literatura com duas formas de cálculo como apresentados nas Equações 1 e 2.

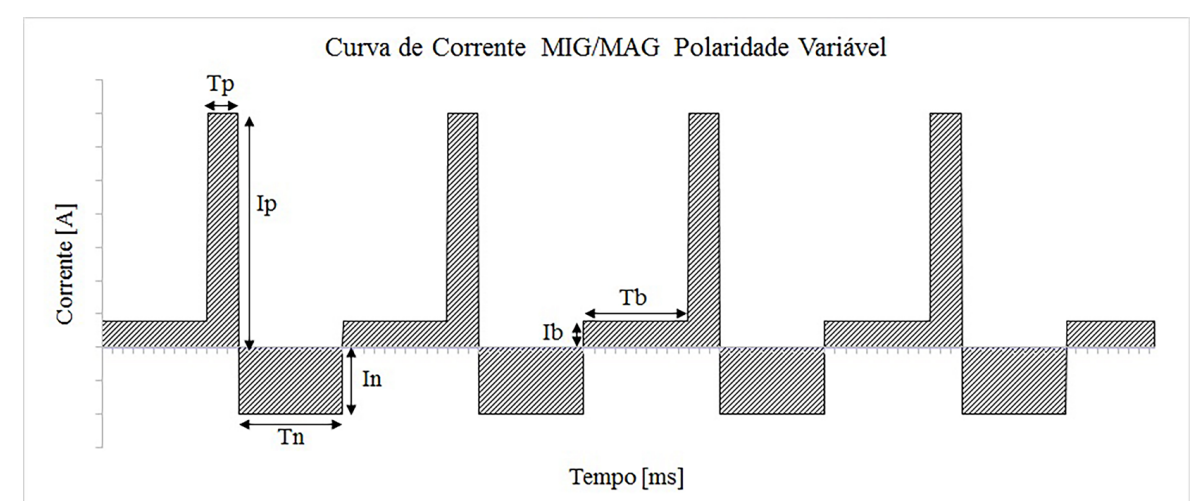

Figura 1. Demonstração dos parâmetros da curva de corrente no processo MIG/MAG PV.

$$
\begin{aligned}
& \% E N=\frac{I n \times t n}{(I n \times T n)+(I p \times T p)+(I b \times T b)} 100 \% \\
& \% E N^{\prime}=\frac{T n}{T n+T p+T b} 100 \%
\end{aligned}
$$

$\mathrm{Na}$ Equação 1 são consideradas as intensidades das correntes positivo e negativo, e os respectivos tempos de aplicação no cálculo. Entretanto, na Equação 2 apenas considera-se o tempo em que se aplica a corrente positiva e negativa, gerando valores diferentes de \%EN para o mesmo conjunto dos seis parâmetros. As duas formas de cálculo evidenciam as diferenças e o possível aumento do eletrodo negativo no processo de soldagem, porém as duas variáveis não são comparáveis entre si. Neste trabalho serão analisados os parâmetros da curva de corrente e não o termo isoladamente, buscando o entendimento individual de cada parâmetro em relação à diluição dos cordões de solda. 
O termo \%EN foi observado por Tong et al. [3], nos experimentos realizando cordões de solda com eletrodo e metal base de alumínio, verificaram que, com o aumento do percentual de eletrodo negativo ocorria redução da penetração no metal base. Esses resultados também são apresentados por Kim et al. [7], onde o incremento da utilização do eletrodo negativo na curva de corrente gerou cordões de solda com menores penetrações. Por fim, Talkington [8], também verificou a redução da penetração, do mesmo modo concluiu que houve uma redução de energia de soldagem, além da diminuição da largura e diluição, e aumento do reforço dos cordões de solda com maiores participações da polaridade negativa (\%EN).

Segundo Puhl [6], o processo de soldagem MIG/MAG com polaridade variável pode ser empregado na recuperação de peças e dutos que sofreram algum tipo de desgaste, como por exemplo, o efeito da corrosão. Em soldas para revestimentos de dutos, em geral, há necessidade de soldar em operação, apresenta benefícios como altas velocidades de soldagem com menor aporte térmico e por consequência menores distorções da junta soldada.

As soldas para revestimento e preenchimento, segundo Gomes [9], estão sendo utilizadas com maior frequência no ambiente industrial, devido à alta produtividade do processo aliada à possibilidade de produzir peças, componentes, estruturas oriundas de materiais de baixo custo e após a solda para revestimento, essa superfície final ganha as propriedades desejadas, por exemplo, anticorrosivas.

Alta confiabilidade, baixos de custo de operação, altas velocidades de soldagem gerando altas taxas de produtividade e estes aliados à fácil operação e possibilidade de soldagem em todas as posições, fazem com que o processo MIG/MAG seja um dos principais processos de soldagem para revestimento e preenchimento, como apresentado por Murugan e Parmar [10]. Os autores também enfatizaram que para atingir ótimos resultados de soldas para revestimentos e preenchimentos com o processo MIG/MAG é necessário trabalhar na otimização dos parâmetros de soldagem objetivando uma minimização da diluição. Uma das aplicações de solda de revestimento é a utilização de aço inoxidável em recobrimento de peças de metal base de aço ao carbono que resultam em superfícies com propriedades com proteção à corrosão.

Conforme apresentado por Gomes [9], a diluição é um dos principais parâmetros para obter excelentes soldas de revestimento em aço inoxidável sobre aços ao carbono. Nesse caso é necessário o controle rigoroso da diluição, buscar minimizar, durante o processo de soldagem, dessa forma reduz a influência do metal base para que o efeito sobre as características do revestimento seja mínimo.

A diluição é a razão entre a área do metal base fundido, neste caso é o aço ao carbono, em relação ao total da área fundida, que seria a soma das áreas transversais do metal base mais o metal de adição, que neste caso é o aço inoxidável, conforme Figura 2, e o cálculo da diluição (D) segundo a Equação 3.

$$
\% D=\frac{A}{A+B} \times 100
$$

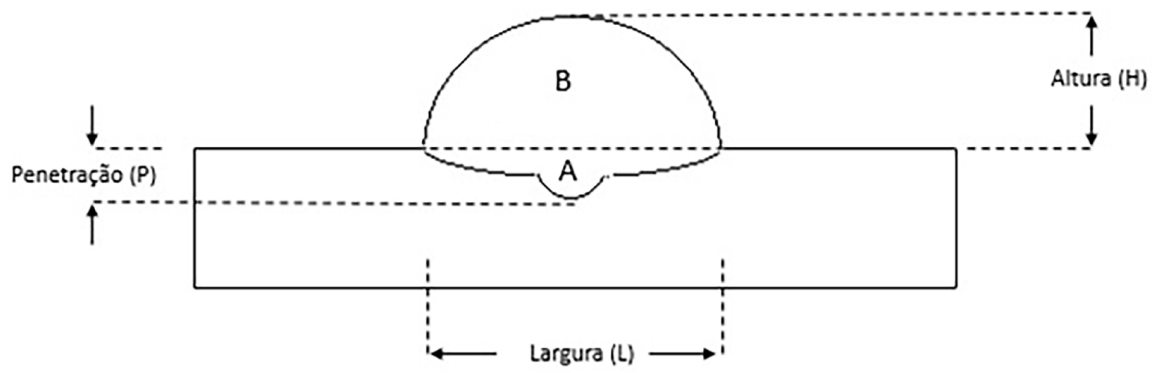

Figura 2. Esquema do cordão de solda livre sobre a chapa (bead-on-plate: BOP) e as áreas usadas na determinação da Diluição. 
Murugan e Parmar [11], para obter cordões de solda para revestimento com qualidade, por exemplo, maximizar a propriedade anticorrosiva do recobrimento, a melhor distribuição para os próximos passes e considerando o aspecto econômico do processo, sugerem valores de diluição entre 10 e 15\%. Além da minimização da diluição, como exposto por Gomes [9], deve-se também: minimizar a penetração e maximizar a altura e largura do cordão de solda, dessa forma é possível garantir as vantagens do processo de soldagem no revestimento e preenchimento.

Considerando os benefícios e características na utilização do MIG/MAG polaridade variável, como baixa penetração e diluição e as características necessárias para as soldas de revestimento e preenchimento, este trabalho tem por objetivo avaliar os parâmetros da polaridade negativa da curva de corrente (corrente negativa e tempo da corrente negativa, e tempo da corrente positiva), visando otimizar a geometria do cordão de solda para aplicação de revestimento com metal base aço ao carbono e metal de adição de aço inoxidável.

\section{Materiais e Métodos}

No experimento foram utilizadas chapas de aço SAE 1020 de 6,35 mm (1/4") de espessura como metal base, metal de adição arame de aço inoxidável ER308LSi de 1,2 mm de diâmetro, e gás de proteção $\mathrm{Ar}+2 \% \mathrm{O}_{2} \mathrm{com}$ vazão de 15 l/min. Todos os cordões de solda foram realizados no Laboratório de Soldagem \& Técnicas Conexas da UFRGS, com a fonte de soldagem Multiprocessos DIGIPlus A7 450 e auxílio de um Tartílope V4 para movimentação da tocha. Foi utilizado o sistema de aquisição portátil SAP 4.01 (IMC Soldagem) para coletar informações de corrente e tensão durante a soldagem nos experimentos (taxa de aquisição de 5000 pontos por segundos, divido em três canais de aquisição). A Figura 3 encontram-se os equipamentos utilizados.

A curva de corrente de polaridade variável usada possui seis parâmetros independentes (Ip, Ib, In, Tp, Tb e Tn). Neste experimento são analisados três parâmetros utilizando a metodologia da superfície de resposta em conjunto com o projeto de experimentos Box-Behnken, variando-se três parâmetros e cada um desses em três níveis. Visando um maior entendimento dos efeitos da polaridade negativa no processo, três parâmetros de controle foram variados e cada um em três níveis, eles são: corrente negativa (In), tempo na polaridade negativa (Tn) e tempo de base positiva (Tb), a Tabela 1 mostra os valores dos níveis empregados.
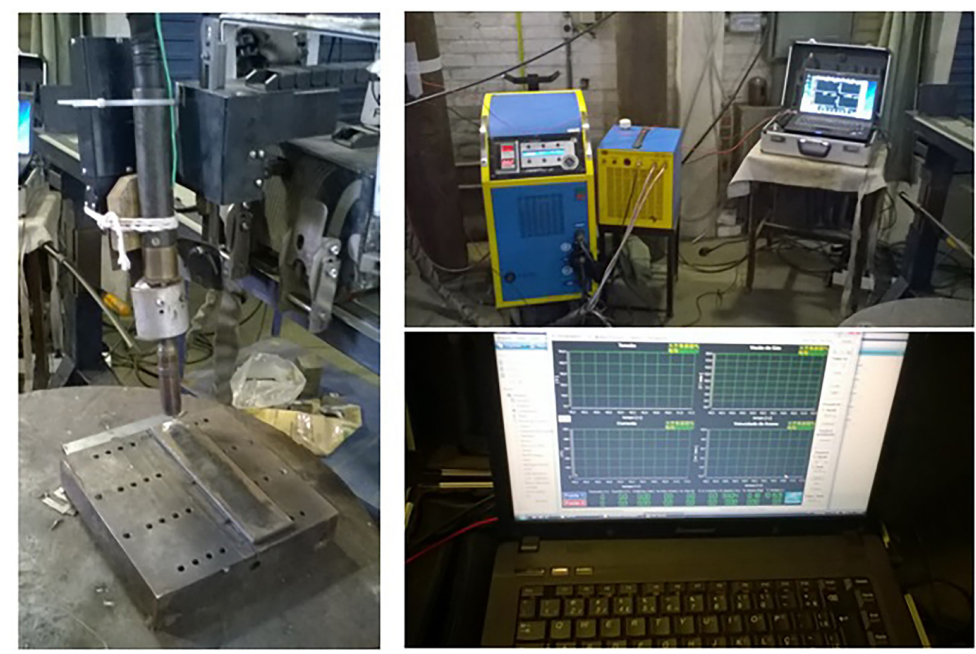

Figura 3. Equipamentos utilizados no experimento.

Tabela 1. Parâmetros independentes e os níveis de variação no projeto de experimentos.

\begin{tabular}{cccc}
\hline Parâmetros & Níveis de Variação & -50 \\
\hline $\ln [\mathrm{A}]: \mathrm{X}_{1}$ & -150 & -100 & 15 \\
\hline $\operatorname{Tn}[\mathrm{ms}]: \mathrm{X}_{2}$ & 5 & 10 & 15 \\
\hline $\operatorname{Tb}[\mathrm{ms}]: \mathrm{X}_{3}$ & 5 & 10 & 15 \\
\hline
\end{tabular}


O projeto de experimentos Box-Behnken é um dos tipos de metodologia de superfície de resposta (MSR), o qual consiste em um projeto com três níveis fatoriais incompletos, que é obtido pela combinação de projetos $2^{2}$ fatoriais blocados que contém todas as combinações dos três fatores [12]. Considerando um experimento com três fatores e três níveis, são realizados 15 experimentos, sendo 12 relacionados às combinações de valores máximos ou mínimos de dois fatores, mais um fator em seu nível médio, e três ensaios considerando a combinação dos valores médios para os três fatores (ponto central). Um dos grandes benefícios do projeto Box-Behnken é seu custo efetivo se comparado com outros projetos, o qual requer um número reduzido de experimentos para obter resultados satisfatórios de otimização [13]. Além disso, o projeto de experimentos Box-Behnken tem como benefício a relação que é gerada através de um modelo de regressão não linear quadrático, conforme a Equação 4, onde $Y$ é a variável resposta, $X_{1}, X_{2}$ e $X_{3}$ os fatores controláveis, $b_{0}$ a intercepção e $b_{1} a b_{33}$ os coeficientes de regressão.

$$
Y=b_{0}+b_{1} X_{1}+b_{2} X_{2}+b_{3} X_{3}+b_{12} X_{1} X_{2}+b_{13} X_{1} X_{3}+b_{23} X_{2} X_{3}+b_{11} X_{1}^{2}+b_{22} X_{2}^{2}+b_{33} X_{3}^{2}
$$

A etapa de planejamento do experimento foi realizada através de um software de estatística, o qual dividiu de forma aleatória as combinações dos níveis dos parâmetros nos 15 ensaios, conforme a Tabela 2.

Os demais parâmetros da curva de corrente (Ip, Tp, Tb) foram mantidos constantes, corrente de pico (Ip) em 350 A, tempo de pico (Tp) em 3 ms e corrente de base (Ib) em 40 A. Os parâmetros do processo de soldagem foram mantidos constantes, sendo eles: velocidade de soldagem (Vs) de $25 \mathrm{~cm} / \mathrm{min}$, distância bico de contato peça (DBCP) de $18 \mathrm{~mm}$, ângulo da tocha -10 (empurrando), o gás de proteção $\mathrm{Ar}+2 \% 02$ com uma vazão de $15 \mathrm{l} / \mathrm{min}$.

Tabela 2. Matriz do planejamento do experimento Box-Behnken.

\begin{tabular}{cccc}
\hline Sequência de ensaios & In [A] & Tn [ms] & Tb [ms] \\
1 (ponto central) & -100 & 10 & 10 \\
\hline 2 & -100 & 15 & 15 \\
\hline 3 & -100 & 5 & 15 \\
\hline 4 & -150 & 10 & 15 \\
\hline 5 & -150 & 15 & 15 \\
\hline 6 & -50 & 10 & 10 \\
\hline 7 & -50 & 5 & 5 \\
\hline 8 & -50 & 10 & 5 \\
\hline 9 & -100 & 15 & 10 \\
\hline 10 & -100 & 5 & 5 \\
\hline 11 (ponto central) & -100 & 10 & 10 \\
\hline 12 & -150 & 10 & 10 \\
\hline 14 & -100 & 10 & 10 \\
\hline
\end{tabular}

A velocidade de alimentação do arame foi ajustada entre os experimentos com objetivo de manter a tensão de soldagem aproximadamente a $22 \mathrm{~V}$.

Uma vez realizado os cordões de solda de acordo com a sequência da Tabela 2, foram retiradas amostras da seção transversal de cada cordão de solda, dos 15 ensaios, no centro do comprimento dos cordões. Posteriormente, as amostras foram lixadas até obter a superfície apta para realizar o ataque químico com Nital $2 \%$. A partir da imagem de cada macrografia do cordão de solda, áreas A e B foram medidas com a utilização do programa Image J. Como exemplo, na Figura 4 pode-se verificar a macrografia do primeiro ensaio.

Buscando-se otimizar os parâmetros para atingir melhores resultados em soldas aplicadas a revestimento de aço inoxidável sobre aço ao carbono, as variáveis respostas definidas foram: área do metal de adição (B) e área fundida do metal base (A). Na Tabela 3 encontra-se os critérios objetivos necessários para a otimização da característica desejada. 


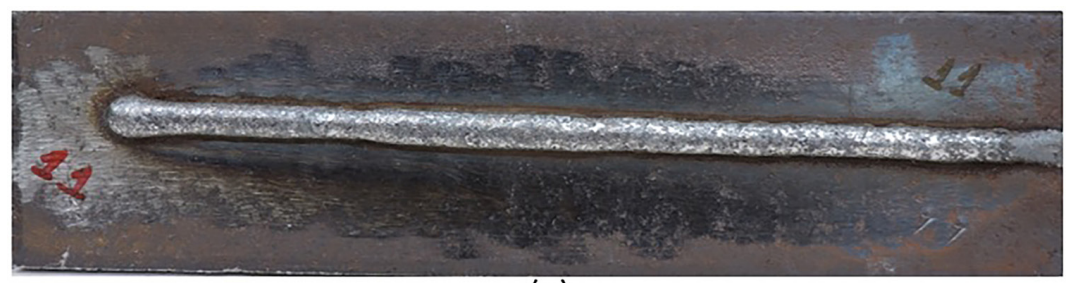

(a)

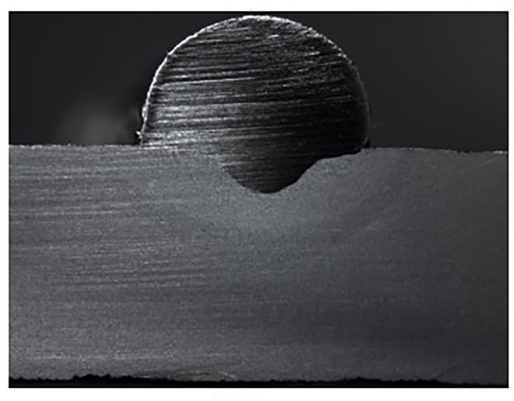

(b)

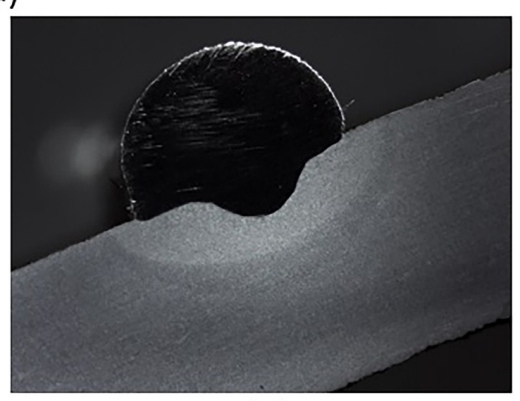

(c)

Figura 4. (a) Cordão de Solda Ensaio \#1 (corpo de prova \#11); (b, c) macrografias Ensaio \#1.

Tabela 3. Variáveis resposta.

\begin{tabular}{cc}
\hline Variável Resposta & Objetivo / alvo \\
A: Área A $\left[\mathrm{mm}^{2}\right]$ & Minimizar \\
\hline B: Área B $\left[\mathrm{mm}^{2}\right]$ & Maximizar \\
\hline
\end{tabular}

\section{Resultados e Discussão}

Primeiramente foram realizadas as medições das macrografias das seções transversais de todos os corpos de prova, medindo as áreas A e B para posterior cálculo da diluição, conforme a Equação 3. Os resultados encontram-se na Tabela 4.

Os resultados da diluição encontram-se na Figura 5, junto com os limites propostos por Murugan e Parmar [11], entre 10 e $15 \%$ de diluição para obter soldas para revestimento com qualidade e maximizar as características

Tabela 4. Resultados da geometria dos cordões de solda dos ensaios do experimento.

\begin{tabular}{ccccccc}
\hline$\#$ & Tb $(\mathbf{m s})$ & Tn $(\mathbf{m s})$ & In $(\mathbf{A})$ & Área $\mathbf{A}\left(\mathbf{m m}^{2}\right)$ & Área $\left.\mathbf{B}(\mathbf{m m})^{2}\right)$ & Diluição $(\%)$ \\
\hline 1 & 10 & 10 & -100 & 3,24 & 18,29 & 15,05 \\
\hline 2 & 15 & 15 & -100 & 2,65 & 15,87 & 14,3 \\
\hline 3 & 15 & 5 & -100 & 3,87 & 14,64 & 20,89 \\
\hline 4 & 15 & 10 & -150 & 3,43 & 19,68 & 14,85 \\
\hline 5 & 10 & 15 & -150 & 3,38 & 25,12 & 11,86 \\
\hline 6 & 15 & 10 & -50 & 2,12 & 12,58 & 14,44 \\
\hline 7 & 10 & 5 & -50 & 3,57 & 15,13 & 19,09 \\
\hline 8 & 5 & 10 & -50 & 2,84 & 14,33 & 16,56 \\
\hline 9 & 5 & 15 & -100 & 3,32 & 18,63 & 15,14 \\
\hline 10 & 5 & 5 & -100 & 4,82 & 20,18 & 19,3 \\
\hline 11 & 10 & 10 & -100 & 3,29 & 17,71 & 15,67 \\
\hline 12 & 5 & 10 & -150 & 4,77 & 25,38 & 15,83 \\
\hline 13 & 10 & 10 & -100 & 2,96 & 17,39 & 14,52 \\
\hline 14 & 10 & 5 & -150 & 4,24 & 20,82 & 16,92 \\
\hline 15 & 10 & 15 & -50 & 1,9 & 13,3 & 12,52 \\
\hline
\end{tabular}


anticorrosivas do metal de solda da superfície soldada. Os resultados obtidos apresentaram 8 dos 15 ensaios entre os limites propostos e o restante dos 7 ensaios acima de $15 \%$. Tendo como maior valor obtido de diluição de $20,9 \%$ no ensaio 3 . Nenhum ensaio apresentou valores de diluição inferiores a $10 \%$.

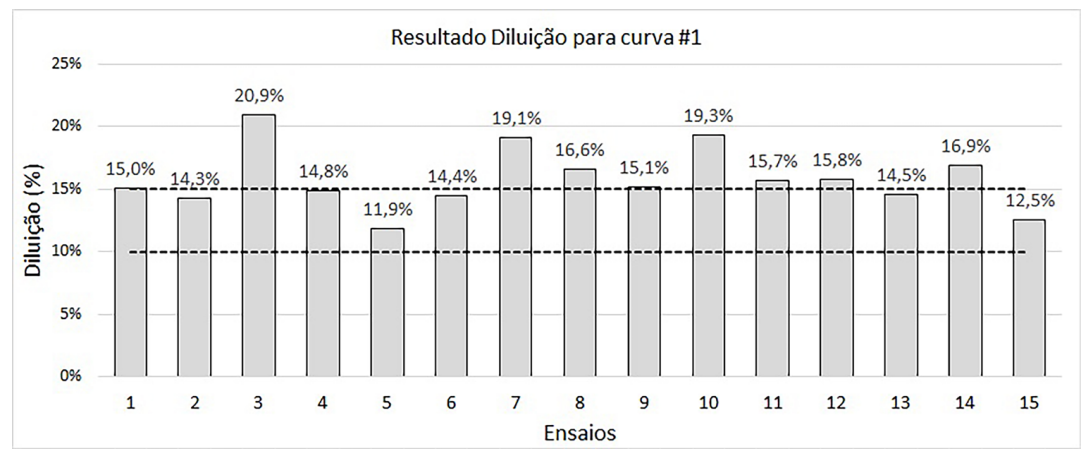

Figura 5. Resultado da Diluição (\%) para cada combinação de parâmetros da Tabela 4.

Para avaliar o efeito dos parâmetros principais e suas interações sobre a diluição, primeiramente foi realizada análises de variância (ANOVA) para cada uma das variáveis resposta, para a área $A$, e após a área B. É muito útil expressar os resultados de um experimento planejado em termos de um modelo de regressão, que facilita a interpretação do experimento. O modelo de regressão polinomial de segundo grau é usado para gerar gráficos, em geral, denominado de Metodologia da Superfície de Resposta (MSR).

A metodologia da superfície de resposta é um conjunto de técnicas matemáticas e estatísticas úteis na modelagem e na análise em aplicações em que a resposta de interesse é afetada por diversos parâmetros (ou variáveis independentes) e o objetivo é otimizar a resposta.

Através do software Minitab ${ }^{\circledR}$, os dados da Tabela 4 foram processados para, então, realizar-se a análise de variância e determinar a influência dos fatores e/ou interações sobre as respostas, área A e área B. Para essa análise foi utilizado um intervalo de confiança de $95 \%$, ou seja, para o valor-p menor que $5 \%$, admitiu-se que a variável de controle em questão é significativa na resposta. Vale ressaltar que, quanto menor for o valor-p, maior será a influência do parâmetro na resposta analisada.

Da ANOVA, Tabela 5, pode ser determinado que os três parâmetros principais, corrente negativa, tempo de corrente negativa e tempo de base positiva, são significativos (valor- $p<0,05$ ) sobre a área $A$. Nenhuma interação entre os fatores é significativa (valor-p >0,05).

Tabela 5. Análise de Variância dos parâmetros $I_{n^{\prime}}, T_{n}$ e $I_{p}$ sobre a área $A$.

\begin{tabular}{|c|c|c|c|c|c|c|}
\hline Fator & SQ & GDL & QM & $\mathbf{F}$ & Valor-p & Significância \\
\hline$I_{n}[A](L)$ & 3,624778 & 1 & 3,624778 & 110,9909 & 0,008890 & Significativo \\
\hline$I_{n}[A](Q)$ & 0,063323 & 1 & 0,063323 & 1,9390 & 0,298393 & Não-Signif. \\
\hline$T_{n}[\mathrm{~ms}](\mathrm{L})$ & 3,438753 & 1 & 3,438753 & 105,2948 & 0,009364 & Significativo \\
\hline$T_{n}[m s](Q)$ & 0,217205 & 1 & 0,217205 & 6,6508 & 0,123183 & Não-Signif. \\
\hline$T_{b}[\mathrm{~ms}](\mathrm{L})$ & 1,705705 & 1 & 1,705705 & 52,2288 & 0,018614 & Significativo \\
\hline $\mathrm{T}_{\mathrm{b}}[\mathrm{ms}](\mathrm{Q})$ & 0,253052 & 1 & 0,253052 & 7,7485 & 0,108462 & Não-Signif. \\
\hline $\mathrm{I}_{n}(\mathrm{~L}) \operatorname{por}_{\mathrm{n}}(\mathrm{L})$ & 0,162006 & 1 & 0,162006 & 4,9606 & 0,155802 & Não-Signif. \\
\hline $\mathrm{I}_{\mathrm{n}}(\mathrm{L}) \operatorname{por}_{\mathrm{b}}(\mathrm{L})$ & 0,097344 & 1 & 0,097344 & 2,9807 & 0,226406 & Não-Signif. \\
\hline$T_{n}(L) \operatorname{por}_{b}(L)$ & 0,019881 & 1 & 0,019881 & 0,6088 & 0,516935 & Não-Signif. \\
\hline Falta de ajuste & 0,177551 & 3 & 0,059184 & 1,8122 & 0,374928 & \\
\hline Erro Puro & 0,065317 & 2 & 0,032658 & & & \\
\hline Total & 9,832204 & 14 & & & & \\
\hline
\end{tabular}


O coeficiente de determinação $\mathrm{R}^{2}$ é uma medida de ajuste de um modelo estatístico generalizado. $\mathrm{O}$ valor de $\mathrm{R}^{2}$ é obtido pela comparação entre os valores obtidos a partir dos dados experimentais e os valores previstos pelo modelo. A análise estatística indica que o modelo matemático é adequado, resultando um $R^{2}=0,9753$. Na Figura 6 se mostra a proporção que pode ser interpretada como o efeito da variação do parâmetro ou interação sobre a resposta (área A). Há grande influência dos fatores da polaridade negativa, a corrente negativa (In, $\approx 37 \%$ ) e o tempo de corrente negativa $(\mathrm{Tn}, \approx 35 \%)$ e o tempo da corrente de base $(\mathrm{Tb}, \approx 17 \%$ ) sobre a área $\mathrm{A}$.

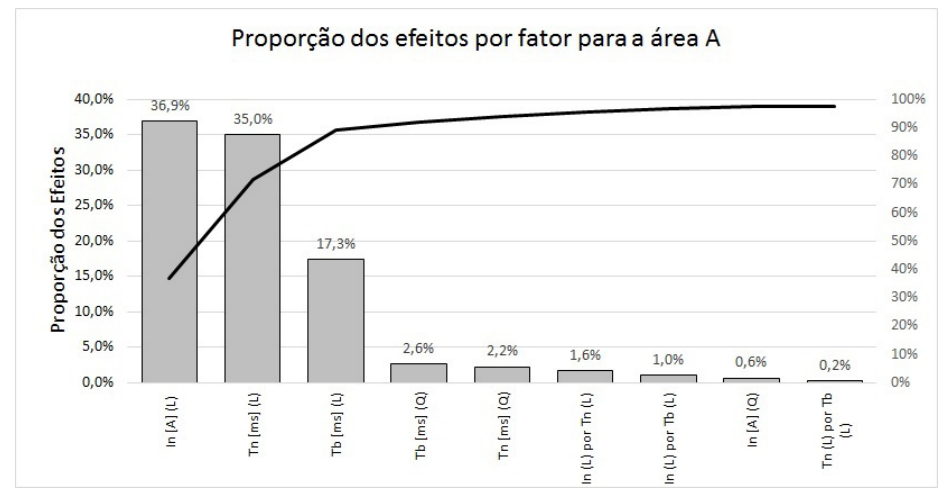

Figura 6. Estimativa da proporção dos efeitos por fator sobre a área A.

Na Figura 7 se apresenta o gráfico dos efeitos dos parâmetros principais sobre a área $A$. Nesse gráfico pode ser observada a grande parcela do efeito da corrente negativa sobre a área $A$, assim como, com o aumento do valor absoluto da corrente negativa ( $\mathrm{In})$, aumentando de $-50 \mathrm{~A}$ para -150 A, resulta em um grande aumento do valor da área $A\left(\mathrm{de} \approx 2,60 \mathrm{~mm}^{2}\right.$ para $\approx 4 \mathrm{~mm}^{2}$ ). Porém para o tempo de corrente negativa e o tempo de base, para incrementos nos valores de tempo (5 para $15 \mathrm{~ms}$ ) causam redução na área $A$.

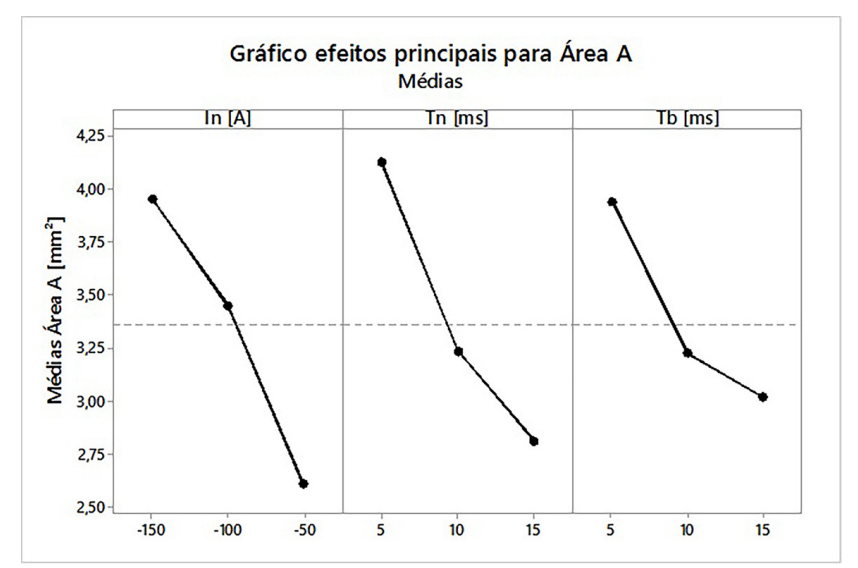

Figura 7. Gráfico dos efeitos dos parâmetros principais sobre a área A.

As superfícies de resposta, na Figura 8, estão considerando os fatores da polaridade negativa (In e Tn) e mantendo constante o tempo de base (Tb) em 10 e 15 ms, os quais, segundo a Figura 7, apresentaram menores valores da área A. Considerando os dois gráficos da superfície de resposta, os valores mínimos da área $A$ (na região em verde área $A<2 \mathrm{~mm}^{2}$ ), pode selecionar-se a combinação de $\mathrm{ln}=-50 \mathrm{~A}$ e Tn $=15 \mathrm{~ms}$. Analisando a energia teórica de soldagem envolvida entre as duas combinações de parâmetros, a combinação de In = -50 A, Tn = $15 \mathrm{~ms}$ e Tb = $15 \mathrm{~ms}$ resulta em um valor teórico de energia de soldagem aproximado de $600 \mathrm{~J} / \mathrm{mm}$. Entretanto para In = $-50 \mathrm{~A}, \mathrm{Tn}=15 \mathrm{~ms}$ e alterando para $\mathrm{Tb}=10 \mathrm{~ms}$, o valor teórico de energia de soldagem aumenta para aproximados $650 \mathrm{~J} / \mathrm{mm}$, maior energia de soldagem para um menor tempo de corrente de base (10 ms), onde a peça por sua vez recebe maior energia, em consequência causa um aumento na área fundida do metal base. 


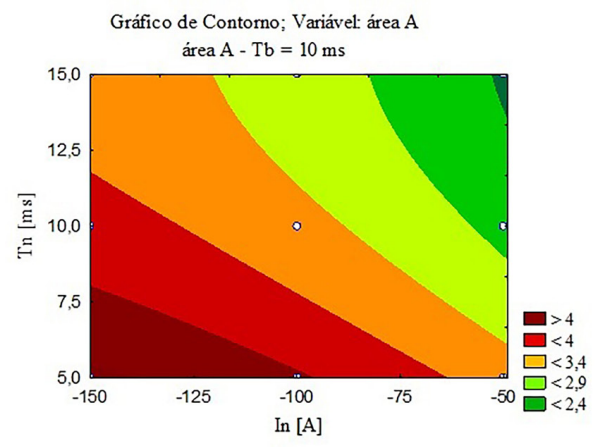

(a)

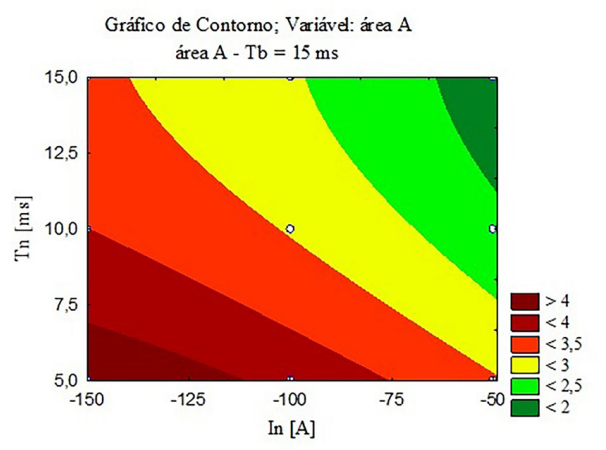

(b)

Figura 8. Gráfico de Contorno da área A em relação a In e Tn com Tb = 10 ms (a); e com Tb = 15 ms (b).

Em sequência, para análise da diluição do processo, necessita-se além da área $A$, o estudo da área $B$, seção transversal da área do metal de adição. A Tabela 6 mostra a análise de variância (ANOVA) dos parâmetros In, Tn e Tb sobre a área $B$ onde os parâmetros da corrente negativa (In) e tempo de base (Tb) mostram-se significativos. Considerando a proporção, efeito de cada fator sobre a área B (Figura 9), a variação da corrente negativa (In - A) representa $75,5 \%$ do total da variação dos efeitos sobre a área B, seguido do tempo de base ( $\mathrm{Tb}-\mathrm{ms}$ ) com $14,7 \%$. Para a área B duas interações são significativas, a interação entre a corrente negativa e o tempo de corrente negativa (In por Tn) com 4,5\% da proporção, em seguida, a interação entre a corrente negativa e o tempo de base positiva (In por Tb), com 1,9\% da proporção.

Tabela 6. Análise de Variância dos parâmetros $I_{n^{\prime}} T_{n}$ e $I_{p}$ sobre a área $B$.

\begin{tabular}{|c|c|c|c|c|c|c|}
\hline Fator & SQ & GDL & QM & $\mathbf{F}$ & Valor-p & Significância \\
\hline$I_{n}[A](L)$ & 159,0704 & 1 & 159,0704 & 762,1439 & 0,001310 & Significativo \\
\hline $\mathrm{I}_{\mathrm{n}}[\mathrm{A}](\mathrm{Q})$ & 1,9531 & 1 & 1,9531 & 9,3576 & 0,092307 & Não-Signif. \\
\hline $\mathrm{T}_{\mathrm{n}}[\mathrm{ms}](\mathrm{L})$ & 0,5800 & 1 & 0,5800 & 2,7787 & 0,237451 & Não-Signif. \\
\hline $\mathrm{T}_{\mathrm{n}}[\mathrm{ms}](\mathrm{Q})$ & 0,0163 & 1 & 0,0163 & 0,0783 & 0,805863 & Não-Signif. \\
\hline$T_{b}[\mathrm{~ms}](\mathrm{L})$ & 30,9881 & 1 & 30,9881 & 148,4715 & 0,006668 & Significativo \\
\hline $\mathrm{T}_{\mathrm{b}}[\mathrm{ms}](\mathrm{Q})$ & 1,0596 & 1 & 1,0596 & 5,0769 & 0,153010 & Não-Signif. \\
\hline$I_{n}(L)$ por $T_{n}(L)$ & 9,4004 & 1 & 9,4004 & 45,0393 & 0,021490 & Significativo \\
\hline$I_{n}(L)$ por $T_{b}(L)$ & 3,9105 & 1 & 3,9105 & 18,7362 & 0,049447 & Significativo \\
\hline$T_{n}(L) \operatorname{por} T_{b}(L)$ & 1,9321 & 1 & 1,9321 & 9,2572 & 0,093173 & Não-Signif. \\
\hline Falta de ajuste & 1,1074 & 3 & 0,3691 & 1,7687 & 0,381086 & \\
\hline Erro Puro & 0,4174 & 2 & 0,2087 & & & \\
\hline Total & 210,67 & 14 & & & & \\
\hline
\end{tabular}

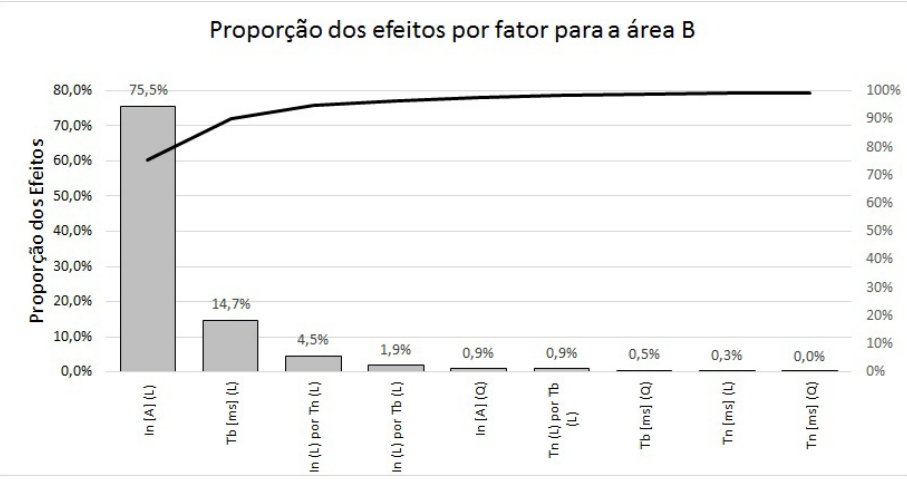

Figura 9. Gráfico dos efeitos dos parâmetros principais sobre a área B. 
O valor de $\mathrm{R}^{2}$ é obtido pela comparação entre os valores obtidos a partir dos dados experimentais e os valores previstos pelo modelo. A análise estatística indica que o modelo matemático é adequado, resultando um $R^{2}=0,9928$.

O tempo da corrente negativa não teve efeito significativo, onde foi verificado através da proporção dos efeitos de apenas 0,3\% sobre a resposta área B. Na Figura 10 observa-se que para variações de Tn (entre 5 e 15 ms), o valor médio da área B não se altera consideravelmente (se mantém em torno do valor médio de 18 mm²).

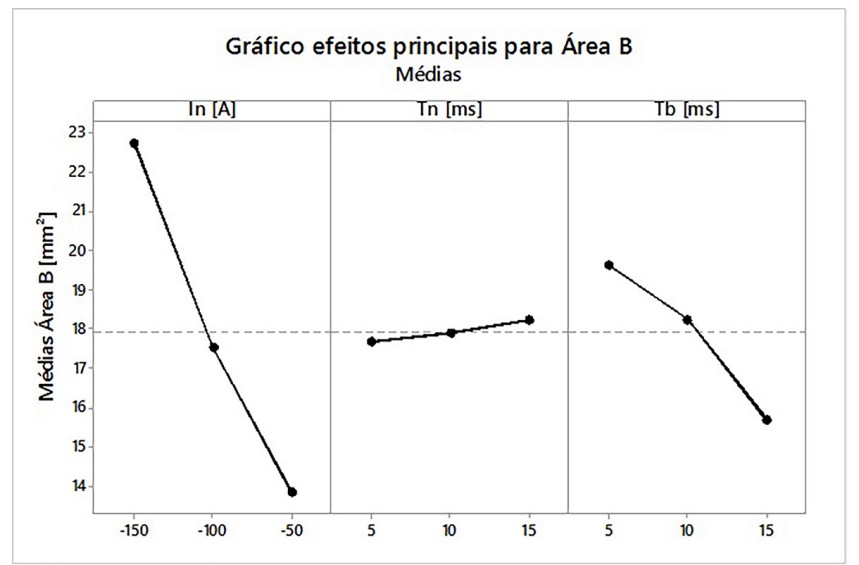

Figura 10. Gráfico dos efeitos principais para a área B.

Considerando os efeitos individuais dos três parâmetros avaliados (Figura 10) pode-se comprovar a grande parcela da corrente negativa (In - A) sobre a variável resposta, quando a corrente negativa é variada em -150 A, essa apresenta os maiores valores de área $B \approx 23 \mathrm{~mm}^{2}$, assim como, o tempo de base ( $\mathrm{Tb}-\mathrm{ms}$ ) quando considerado em seu nível inferior de $5 \mathrm{~ms}$, assim encontram-se os maiores valores da área do metal de adição $\left(\mathrm{B} \approx 20 \mathrm{~mm} \mathrm{~m}^{2}\right.$ ). E observa-se que o efeito individual do tempo de corrente negativa ( Tn) não é significativo, variando de 5 para 15 ms, apresenta uma pequena variação da área B.

A significância das interações determinada pela ANOVA (Tabela 6) pode ser comprovada mediante o gráfico de interação de dois fatores, conforme a Figura 11. Graficamente a interação é significativa quando há cruzamentos entre as linhas gráficas dos diferentes níveis de algum fator, como é o caso apresentado na Figura 11a, no gráfico entre In x Tn. Para os valores de In = -150 A e Tn = 15 ms é possível obter a maior área $B \approx 25 \mathrm{~mm}^{2}$. Se In for mantido constante ( $\mathrm{In}=-150 \mathrm{~A}$ ) e reduzindo o valor de Tn causa diminuição na área $\mathrm{B}$, por exemplo, quando $\mathrm{Tn}=10 \mathrm{~ms}$ a área $\mathrm{B} \approx 23 \mathrm{~mm}^{2}$ e ao ajustar $\mathrm{Tn}(=5 \mathrm{~ms})$ ao valor mínimo a área $\mathrm{B}\left(\approx 21 \mathrm{~mm}^{2}\right)$ obtida é mínima. A corrente negativa no nível intermediário ( $\mathrm{In}=-100 \mathrm{~A})$ observa-se que a área $\mathrm{B}\left(\approx 18 \mathrm{~mm}^{2}\right)$ é independente do valor de Tn, isto é, pode

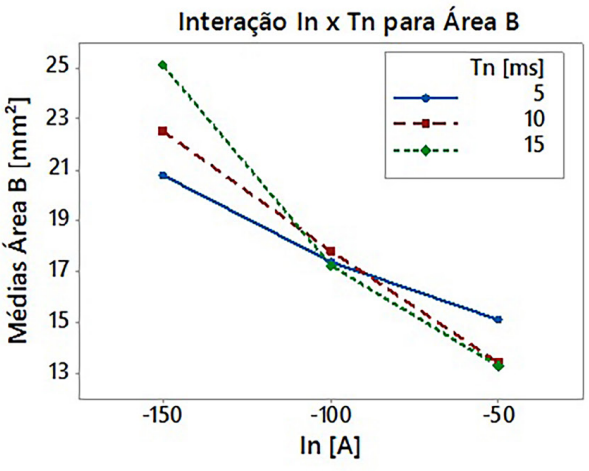

(a)

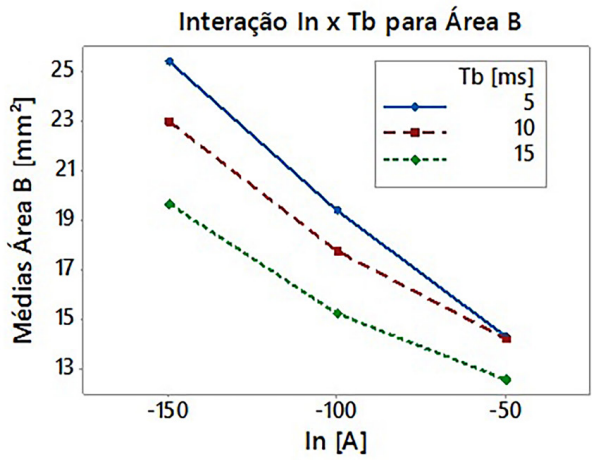

(b)

Figura 11. Gráfico da interação dos fatores para a área B em relação a In e Tn (a); e área B em relação a In e Tb (b). 
ser ajustado no valor de 5, 10 ou $15 \mathrm{~ms}$. Entretanto, usando In = -50 A e Tn = $15 \mathrm{~ms}$ ou $10 \mathrm{~ms}, \mathrm{a}$ área $\mathrm{B} \approx 13 \mathrm{~mm}$. A área $B \approx 15 \mathrm{~mm}^{2}$ para a $\mathrm{In}=-50 \mathrm{~A}$ e $\mathrm{Tn}=5 \mathrm{~ms}$.

Visando valores entre $10 \%$ a $15 \%$ de diluição, os parâmetros principais de controle (In, Tn e Tb) devem ser combinados para maximizar os valores de área $\mathrm{B}$, considerando a equação da diluição. Verificando o gráfico dos efeitos dos parâmetros principais (Figura 10), assim como o gráfico da interação de dois fatores (Figura 11), os valores para a maximização da área de metal de adição seriam, corrente negativa a In = $-150 \mathrm{~A}$, tempo de base $\mathrm{Tb}=5 \mathrm{~ms}$. Para um melhor entendimento dos efeitos dos níveis do tempo de corrente negativa ( $\mathrm{Tn}$ ), assim como sua interação com a corrente negativa (In), foi realizado um gráfico de contorno da superfície de resposta, conforme Figura 12.

Com o auxílio dos gráficos de contorno das superfícies de resposta (Figura 12), foi verificado uma leve influência do tempo de corrente negativa (Tn) sobre a variável resposta, onde, para atingir valores máximos da área $\mathrm{B}$, o valor de Tn deve ser ajustado em 15 ms. Embora a energia teórica de soldagem considerando a combinação dos parâmetros de $\mathrm{Tn}=5 \mathrm{~ms}$, Tb = $5 \mathrm{~ms}$ e In = $-150 \mathrm{~A}$, resulte em aproximados $1025 \mathrm{~J} / \mathrm{mm}$, e considerando $\mathrm{Tn}=15 \mathrm{~ms}$, em torno de $930 \mathrm{~J} / \mathrm{mm}$. Para maximizar a área B usando maiores valores de tempo de corrente negativa, pode ser explicado que incrementando a ação da polaridade negativa causa um aumento na taxa de fusão e redução no aporte térmico, conforme apresentado por Monteiro e Scotti [14].

Em suma, para maximizar a área $\mathrm{B}$, a combinação dos parâmetros necessária é: $\mathrm{Tn}=15 \mathrm{~ms}, \mathrm{~Tb}=5 \mathrm{~ms} \mathrm{e}$ In $=-150 \mathrm{~A}$.

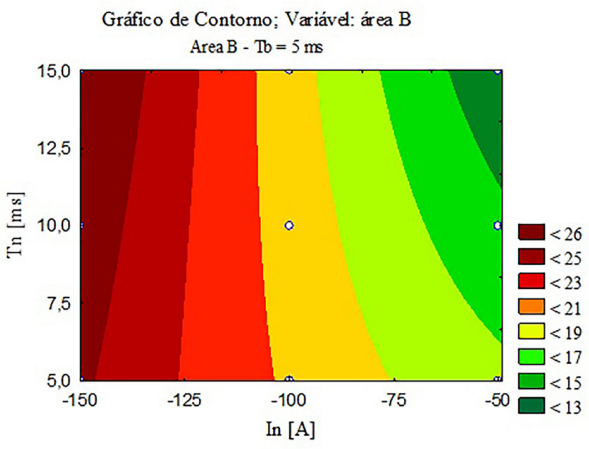

(a)

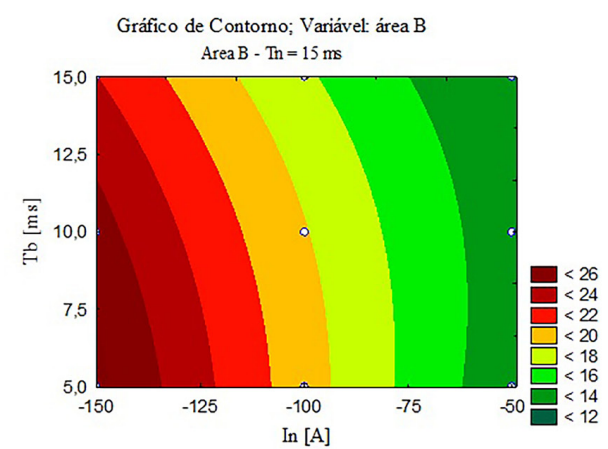

(b)

Figura 12. Gráfico de Contorno da área B em relação a In e Tn com Tb = 5 ms (a); e área B em relação a In e Tb com $\mathrm{Tn}=15 \mathrm{~ms}$ (b).

Na Figura 13 são apresentadas a relação entre os valores observados e os valores estimados tanto para a área $A$, quanto para a área B. Os valores estimados usando o modelo de regressão (Equações 5 e 6), para a área A e área $B$, em função dos valores dos parâmetros avaliados. Quanto mais os pontos se aproximam da reta de referência, melhor é o ajuste do modelo (valores estimados) aos dados (valores observados).

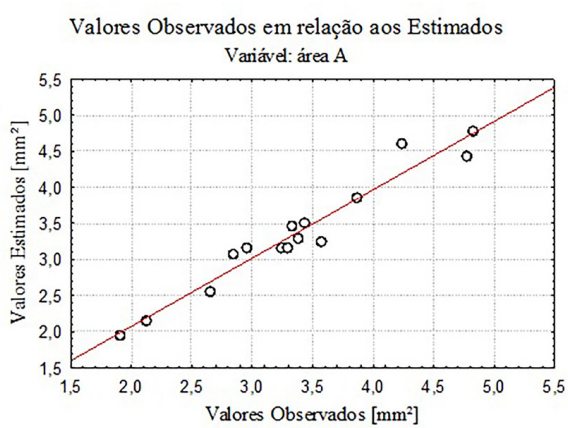

(a)

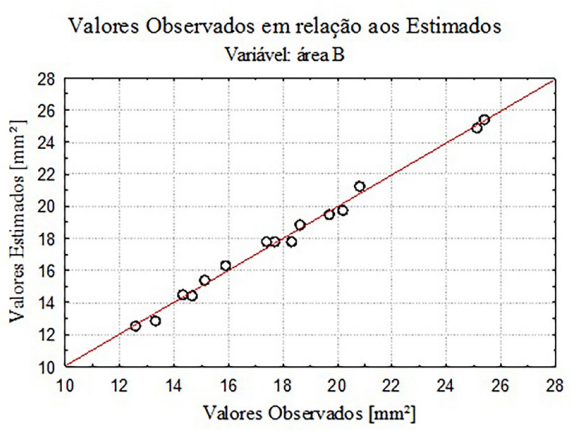

(b)

Figura 13. Gráfico comparativo entre valores observados e valores estimados para a área A (a); e área B (b). 
Os parâmetros necessários para minimizar a área fundida do metal base (área A) e maximizar a área do metal de adição (área B), são apresentados na Tabela 7.

Tabela 7. Níveis necessários para otimização da área A e área B.

\begin{tabular}{cccc}
\hline & & Fatores & \\
\cline { 2 - 4 } Variável Resposta: Critério & $\ln [\mathbf{A}]$ & Tn $[\mathbf{m s}]$ & Tb $[\mathbf{m s}]$ \\
A: Área A $\left[\mathrm{mm}^{2}\right]$ : Minimizar & -50 & 15 & 15 \\
\hline B: Área B $\left[\mathrm{mm}^{2}\right]$ : Maximizar & -150 & 15 & 5 \\
\hline
\end{tabular}

Como o objetivo é obter as duas respostas simultaneamente, há necessidade de determinar a combinação de parâmetros que aplicado no processo de soldagem para revestimento, forneça o valor-alvo desejado (diluição entre 10 e 15\%). Porém, não há uma mesma combinação de parâmetros que, simultaneamente, possa minimizar a área $\mathrm{A}$ e maximizar a área B, podendo assim chegar ao objetivo de otimização, minimizar a diluição.

A utilização de gráficos de superposição da superfície de resposta das áreas A e B não foi possível devido a dois dos três fatores (In e Tb) apresentarem níveis distintos na sua otimização da variável resposta individual. Por exemplo, na minimização da área A o tempo de base deve ser de 15 ms (nível superior), entretanto, para maximizar a área $B, T$ deve ser ajustado no valor de 5 ms (nível inferior).

Para minimizar o valor a diluição buscou-se a otimização da equação objetivo, composta pelas equações de regressão para área $A$ (Equação 5) e área B (Equação 6) no formato da equação da diluição (Equação 3). Essa equação objetivo (Equação 7), foi resolvida através de rotinas de pesquisa operacional buscando sua minimização, conforme procedimento apresentado por Hillier e Lieberman [15]. Os valores resultantes encontram-se na Tabela 8.

Tabela 8. Valores dos parâmetros principais In, Tn e Tb para minimizar a diluição.

\begin{tabular}{cccc}
\hline & & Fatores \\
\cline { 2 - 4 } Variável Resposta: Critério & $\ln [\mathbf{A}]$ & Tn [ms] & Tb [ms] \\
\cline { 2 - 4 } D: Diluição [\%]: Minimizar & -50 & 15 & 11,50 \\
\hline
\end{tabular}

$$
\begin{aligned}
& \text { ÁreaA }=6,007-0,022 \cdot I_{n}-0,434 \cdot T_{n}-0,268 \cdot T_{b}-0,0008 \cdot I_{n} \cdot T_{n}+0,0006 \cdot I_{n} \cdot T_{b} \\
& +0,003 \cdot T_{n} \cdot T_{b}-0,00005 \cdot I_{n}^{2}+0,010 \cdot T_{n}^{2}+0,010 \cdot T_{b}^{2} \\
& \text { ÁreaB }=18,268-0,009 \cdot I_{n}-0,891 \cdot T_{n}+0,003 \cdot T_{b}-0,006 \cdot I_{n} \cdot T_{n}+0,004 \cdot I_{n} \cdot T_{b} \\
& +0,028 \cdot T_{n} \cdot T_{b}+0,0003 \cdot I_{n}^{2}+0,003 \cdot T_{n}^{2}+0,152 \cdot T_{b}^{2} \\
& \text { Diluição }=\frac{\text { ÁreaA }}{\text { ÁreaA ÁreaB }}
\end{aligned}
$$

Para os valores de corrente negativa In = -50 A, tempo de corrente negativa de $\mathrm{Tn}=15 \mathrm{~ms}$ e tempo de base de 11,50 ms, utilizando a equação de regressão pode-se chegar a valores de área A igual a 1,69 $\mathrm{mm}^{2} \mathrm{e}$ área $\mathrm{B}$ igual a $12,73 \mathrm{~mm}^{2}$, assim resultando em um valor de diluição de $11,7 \%$. Considerando que os valores anteriormente mencionados de In e Tn foram os níveis estudados ( $\mathrm{n} n=-50 \mathrm{~A}$ ) e ( $\mathrm{Tn}=15 \mathrm{~ms}$ ), respectivamente, porém para o tempo de base, o valor de 11,50 ms não um nível ensaiado, foi realizada uma simulação com as equações de regressão (Equações 5 e 6), com o tempo de base mais próximo ensaiado, de 10 ms. Esta simulação resultou em valores de diluição de $11,9 \%$ (área $A=1,74 \mathrm{~mm}^{2}$ e área $B=12,87 \mathrm{~mm}^{2}$ ). Essa combinação de parâmetros está vinculada ao ensaio 15, os resultados calculados e medidos podem ser comparados na Tabela 9. 
Tabela 9. Comparativo do valor de diluição cálculo e observado.

\begin{tabular}{lccc}
\hline Coeficiente de Regressão & Valor Medido (\%) & Valor Equação (\%) & Diferença \\
Ensaio 15 & 12,52 & 11,91 & $+0,61$ \\
In $=-50 \mathrm{~A}$ & & 112 & \\
\hline
\end{tabular}

\section{Conclusões}

A avalição dos parâmetros da polaridade negativa do processo MIG/MAG com polaridade variável foi realizada mediante a aplicação da metodologia da superfície de resposta, através da deposição de cordões de solda em aço inoxidável sobre aço ao carbono, chegando às seguintes conclusões:

- A metodologia da superfície de resposta se torna uma ferramenta importante para determinar a combinação dos parâmetros necessária para a otimização duas ou mais variáveis de resposta simultaneamente.

- Nesse processo é possível obter diluição de $12 \%$ até $15 \%$, dentro da faixa de valores recomendados para aplicações em revestimento usando o processo de soldagem.

- Por meio da avaliação da área $A$ e da área $B$, foi verificado uma relação significativa do tempo de polaridade negativo com a diluição. A corrente negativa no seu nível inferior (-50 A) apresentou valores menores da área $A$, resultando em um menor valor de diluição, principal objetivo para soldagem de revestimento;

- Para a minimização da diluição desse processo, a combinação mais favorável dos parâmetros necessária encontrada foi: In = -50 A, tempo de corrente negativa de $\mathrm{Tn}=15 \mathrm{~ms}$ e tempo de base de $10 \mathrm{~ms}$.

\section{Agradecimentos}

Os autores agradecem ao Laboratório de Soldagem \& Técnicas Conexas (LS\&TC) da Universidade Federal do Rio Grande do Sul (UFRGS).

\section{Referências}

[1] Nascimento AS, Vilarinho LO. Uma contribuição ao estudo da soldagem MIG-CA. In: Anais do $16^{\circ}$ Simpósio de Pós-graduação em Engenharia Mecânica; 2006 dez 11-13.; Uberlândia, Brasil. Uberlândia: Secretaria do Programa de Pós-Graduação em Engenharia Mecânica; 2006. p. 61-71. [acesso em 14 fev. 2017]. Disponível em: http://www.posgrad.mecanica.ufu.br/posmec/16/

[2] Miranda HC, Ferraresi VA. Identificação da transferência metálica na soldagem MIG/MAG pulsado de aço inoxidável a partir de um sensor óptico. In: Associação Brasileira de Engenharia e Ciências Mecânicas. $2^{\circ}$ Congresso Brasileiro de Engenharia de Fabricação; 2003 maio; Uberlândia, Brasil. Rio de Janeiro: ABCM; 2003. p. 557-566 [acesso em 14 fev. 2017]. Disponível em: http://www.abcm.org.br/anais/cobef/2003/ artigos/COF03_0557.pdf

[3] Tong H, Ueyama T, Harada S, Ushio M. Quality and productivity improvement in aluminum alloy thin sheet welding using alternating current pulsed metal inert gas welding system. Science and Technology of Welding and Joining. 2001;6(4):203208. http://dx.doi.org/10.1179/136217101101538776.

[4] Park HJ, Rhee S, Kang MJ, Kim DC. Joining of steel to aluminum alloy by AC pulse MIG welding. Materials Transactions. 2009;50(9):2314-2317. http://dx.doi.org/10.2320/matertrans. M2009105.

[5] Dutra JC, Silva RHG, Savi BM, Marques C, Orestes EA. New methodology for AC-pulsed GMAW parametrization applied to aluminum shipbuilding. Journal of the Brazilian Society of Mechanical Sciences and Engineering. 2015;38(1):99-107.

[6] Puhl EB. Desenvolvimento de tecnologias no processo MIG/ MAG para aumento de produtividade e melhoria da qualidade mediante o uso da polaridade negativa [dissertação]. Florianópolis: Universidade Federal de Santa Catarina; 2011.

[7] Kim TJ, Joe GJ, Kong HS, Cho SM, Kim CU. The study of variable polarity AC pulse GMA welding system. In: International Conference on Electrical Engineering; 2002 July 7-11; Jeju Island, Korea. Nagoya: Center for Integrated Research in Science and Engineering; 2002. p. 688-691.

[8] Talkington J. Variable polarity gas metal arc welding [dissertation] Ohio: The Ohio State University; 1998

[9] Gomes JH. Análise e otimização da soldagem de revestimento de chapas de aço ABNT 1020 com utilização de arame tubular inoxidável austenítico [dissertação]. Itajubá: Universidade Federal de Itajubá; 2010.

[10] Murugan N, Parmar RS. Effects of MIG process parameters on the geometry of bead in the automatic surfacing of stainless steel. Journal of Materials Processing Technology. 1994;41(4):381-398. http://dx.doi.org/10.1016/0924-0136(94)90003-5.

[11] Murugan N, Parmar RS. Stainless steel cladding deposited by automatic gas metal arc welding. Welding Research Supplement. 1997;10(1):391-400.

[12] Montgomery DC. Design and analysis of experiments. 8. ed. Hoboken: John Wiley \& Sons; 2013.

[13] Liu H, Wang K, Schlindwein W, Li M. Using the Box-Behnken experimental design to optimize operating parameters in pulsed spray fluidized bed granulation. International Journal of Pharmaceutics. 2013;488(2):329-388. http://dx.doi.org/10.1016/j. ijpharm.2013.03.057.

[14] Monteiro LS, Scotti A. A methodology for parametrization of the MIG/MAG CA and its application in servisse repair of pipelines of oil and gas. 22. ed. Ribeirão Preto: COBEM; 2013.

[15] Hillier FS, Lieberman GJ. Introdução à pesquisa operacional. 8. ed. Porto Alegre: McGraw-Hill; 2006. 